\title{
Survivin promotes the invasion of human colon carcinoma cells by regulating the expression of MMP-7
}

\author{
FEI GAO ${ }^{1,3}$, YUQIN ZHANG $^{1}$, FENG YANG $^{2}$, PENG WANG $^{4}$, WENJUN WANG ${ }^{1}$, YAN SU $^{1}$ and WEIREN LUO \\ ${ }^{1}$ Cancer Research Institute, Department of Radiotherapy of Nanfang Hospital, Southern Medical University, \\ Guangzhou, Guangdong 510515; ${ }^{2}$ Secondary Clinical College, Guangdong Medical College, Dongguan, \\ Guangdong 523808; ${ }^{3}$ Department of Gastroenterology, the First Affiliated Hospital of Jinan University, \\ Guangzhou, Guangdong 510630; ${ }^{4}$ Department of Neurosurgery, Guangdong General Hospital, \\ Guangdong Academy of Medical Sciences, Guangzhou, Guangdong 510080, P.R. China
}

Received May 16, 2013; Accepted November 25, 2013

DOI: $10.3892 / \mathrm{mmr} .2014 .1897$

\begin{abstract}
Increased expression levels of survivin are crucial for invasion activity in several types of human cancer, including colon carcinoma. However, the molecular mechanisms whereby survivin regulates cancer invasion have not been completely elucidated. To the best of our knowledge, this study is the first to investigate the role of matrix metalloprotease-7 (MMP-7) in cell invasion that is induced by survivin by using in vitro assays, including western blot, immunofluorescence and qPCR analyses. The results demonstrated that the ectopic expression of survivin significantly promoted the invasive activity of colon carcinoma cells (SW620 and HCT-116) and resulted in increased levels of MMP-7 activation. By contrast, the small interfering RNA (siRNA)-based knockdown of survivin markedly reduced cell migration and led to a dose-dependent decrease in MMP-7 expression levels. Compared with the controls, knockdown of MMP-7 by siRNA in colon carcinoma cells led to reduced invasion ability, whereas no obvious changes were observed when MMP-7 expression was silenced in survivin-overexpressing colon carcinoma cells. These findings demonstrate that MMP-7 is crucial for survivin-mediated invasiveness, suggesting that the survivin-mediated MMP-7 signaling pathway is a potential therapeutic target for the treatment of colon carcinoma.
\end{abstract}

\section{Introduction}

Survivin, a member of the family of inhibitor of apoptosis (IAP) proteins, contains 142 amino acid residues. Survivin

Correspondence to: Mr. Weiren Luo, Cancer Research Institute, Southern Medical University, Guangzhou, Guangdong 510515, P.R. China

E-mail: luoweiren@hotmail.com

Abbreviations: CRC, colorectal cancer; MMP-7, metalloprotease-7; IAP, inhibitor of apoptosis; ECM, extracellular matrix; siRNA, small interfering RNA

Key words: survivin, MMP-7, colon carcinoma, invasion functions as a key regulator of mitosis and programmed cell death by inhibiting apoptosis and promoting cell proliferation (1). Studies have identified that survivin is able to promote the migratory/invasive properties of several types of human cancer, including melanoma, prostate cancer and breast cancer (2-4). Survivin has been demonstrated to regulate the invasion ability of colorectal cancer (CRC) $(5,6)$. However, little is known regarding the functional mechanism of survivin in CRC invasion. A better understanding of the mechanisms by which survivin regulates aggressive cellular behaviors may lead to the production of novel therapeutic targets for colon cancer.

The extracellular matrix (ECM) provides a structural framework to support cells and maintains cell functions by mediating cell-cell or cell-ECM interactions. Degradation of ECM components is mainly controlled by matrix metalloproteinases (MMPs), a large group of enzymes (7). The MMPs comprise a family of zinc-dependent endopeptidases that consist of $>21$ human proteases and are important in carcinogenesis. One of the smallest known members of the MMP family, MMP-7, was first identified as an enzyme of the involuting rat uterus and was subsequently determined to be an important marker in human cancer progression (8). For example, MMP-7 has been reported to be overexpressed in numerous types of human malignancy, including ovarian, prostate, gastric and breast cancer (9-12), and is important in cancer progression as it promotes tumor invasion. Furthermore, the ability of CRC cells to migrate and invade has been demonstrated to correlate with an increased expression of MMP-7 (13-15). In addition, the overexpression of MMP-7 has been reported to contribute to clinically aggressive behaviors and is a significant predictor of patient survival in CRC $(16,17)$. Certain studies have indicated that upregulation of survivin and MMP-7 expression levels correlates with metastatic CRC and is significantly associated with liver metastasis of colon cancer $(18,19)$. However, the functional correlation between survivin and MMP-7 remains largely unknown.

The present study identified an alternative role for survivin in tumor progression. It demonstrates that the increased invasion of colon carcinoma cells that overexpress survivin is mediated by the activation of MMP-7. Furthermore, 
overexpression of survivin enhances MMP-7 expression, whereas silencing endogenous survivin in colon cancer cell lines results in a decreased MMP-7 expression. These findings suggest the molecular mechanism by which survivin enhances cancer invasion. In addition, these data indicate that MMP-7 is a potential mediator of this process.

\section{Materials and methods}

Cell culture. 293FT cells were cultured and maintained in Dulbecco's modified Eagle's medium with $10 \%$ fetal bovine serum, and used as packaging cells for lentivirus production. RPMI-1640 supplemented with $10 \%$ fetal bovine serum was used to separately culture SW620 and HCT-116 cells (American Type Culture Collection, Manassas, VA, USA).

Expression vector construction and viral production. Full-lengthsurvivincDNAwasgenerated byPCR and subcloned into the pReceiver-Lv08 expression vector (Guangzhou Fulengen Co., Ltd., Guangzhou, China). Survivin expression in colon carcinoma cells was ablated using short hairpin RNA (shRNA). Sense (GGACCACCGCAUCUCUACA) and antisense (UGUAGAGAUGCGGUGGUCC) oligonucleotides for the double-stranded cassette were designed and the scrambled sense (ACUACCGUUGUUAUAGGUG) and antisense (CACCUAUAACAACGGUAGU) sequences were used. These oligonucleotides were synthesized and generated by PCR and subcloned into the psi-U6 vector (Guangzhou Fulengen Co., Ltd.). The 293FT cells were transfected with pReceiver-Lv105/survivin, psi-U6/survivin shRNA or the control plasmid pReceiver-Lv105 and psi-U6 using the ViraPower $^{\mathrm{TM}}$ Lentiviral Expression system, the BLOCK-iT ${ }^{\mathrm{TM}}$ Lentiviral RNAi Expression system and Lipofectamine ${ }^{\circledR}$ 2000 (Invitrogen Life Technologies, Carlsbad, CA, USA) according to the manufacturer's instructions. The colon carcinoma cells (SW620 and HCT-116) were infected by serial dilution with the virus-containing medium and the stably-transfected cells were selected using puromycin for one week.

siRNA transfection. A small interfering RNA (siRNA) pool against MMP-7 was purchased from Guangzhou Fulengen Co., Ltd. The siRNA was dissolved in siRNA buffer (Guangzhou Fulengen Co., Ltd.) at a concentration of $100 \mu \mathrm{M}$ and transfected into cells using Lipofectamine 2000. The gene-silencing effect was measured by western blotting $48 \mathrm{~h}$ after transfection.

$q P C R$. To measure the mRNA levels of cell-cycle regulators, total RNA was reverse transcribed using a PrimeScript ${ }^{\mathrm{TM}}$ RT Reagent kit (Takara Bio, Inc., Shiga, Japan). qPCR was performed using SYBR ${ }^{\circledR}$ Premix Ex Taq ${ }^{\mathrm{TM}}$ II (Takara Bio, Inc.) on a StrataGene Mx3005P system. The primer sequences used were as follows: Forward: TTCTCAAGGACCACCGCATC and reverse: GCCAAGTCTGGCTCGTTCTC for survivin; and forward: GAGTGAGCTACAGTGGGAACA and reverse: CTATGACGCGGGAGTTTAACAT for MMP-7. GAPDH was used as an endogenous control. The time points for colon carcinoma cells being transfected with MMP-7-specific siRNA were 12, 24, 48, 72 and $96 \mathrm{~h}$. All samples were normalized to internal controls and fold-changes were calculated using relative quantification $\left(2^{-\Delta \Delta C t}\right)$.

Western blot analysis. Protein was extracted by solubilizing cells in RIPA buffer containing a protease and phosphatase inhibitor cocktail (Keygen Biotech. Co. Ltd., Nanjing, China). Protein concentration was quantitated with Bradford protein assay reagent (Keygen Biotech. Co. Ltd.), and $40 \mu \mathrm{g}$ of total protein was loaded onto $4-12 \%$ polyacrylamide gels, separated and then transferred onto a polyvinylidene difluoride membrane. The membrane was probed with mouse anti-Survivin, MMP-7 (Santa Cruz Biotechnology, Inc., Santa Cruz, CA, USA) and proteins were detected with enhanced chemiluminescence detection reagents (Keygen Biotech. Co. Ltd.). Protein loading was normalized by probing the membrane with anti-GAPDH antibodies.

Immunofluorescence analysis. Cell lines were plated on culture slides (Corning Incorporated, Tewksbury, MA, USA) and, after $24 \mathrm{~h}$, were rinsed with phosphate-buffered saline (PBS) and fixed in methanol-acetone for $30 \mathrm{~min}$ at room temperature. The cells were permeabilized with $0.5 \%$ Triton $\mathrm{X}-100$ for $30 \mathrm{~min}$ and blocked for $45 \mathrm{~min}$ in $10 \%$ bovine serum albumin (Sigma-Aldrich, St. Louis, MO, USA) in PBS, and then incubated with primary monoclonal antibodies in PBS for $2 \mathrm{~h}$ at room temperature. After three washes in PBS, the slides were incubated for $1 \mathrm{~h}$ in the dark with secondary goat anti-mouse antibodies (Invitrogen Life Technologies). After another three washes, the slides were stained with 4',6-diamidino-2-phenylindole (Sigma-Aldrich) for $5 \mathrm{~min}$ to visualize the nuclei and were examined using an Eclipse TE300 microscope (Nikon, Tokyo, Japan).

Boyden chamber assay. Biocoat ${ }^{\mathrm{TM}}$ Growth Factor-Reduced Matrigel $^{\mathrm{TM}}$ invasion chambers $(8.0 \mu \mathrm{m}$ PET membrane; 24-well cell culture inserts; BD Biosciences, Franklin Lakes, NJ, USA) were preincubated in RPMI-1640 supplemented with $1 \%$ fetal bovine serum for $1 \mathrm{~h}$. Harvested cells $\left(2 \times 10^{3}\right.$ cells $)$ in $100 \mu \mathrm{l}$ RPMI-1640 supplemented with $1 \%$ fetal bovine serum were replated onto the upper chamber and placed in the lower chamber with $500 \mu 1$ RPMI-1640 supplemented with $10 \%$ fetal bovine serum. After $20 \mathrm{~h}$, the cells were fixed with methanol. Non-migrated cells on the upper side of the filter were removed with a cotton swab and cells on the underside of the filter were stained with hematoxylin. Images were captured using an Eclipse TE300 microscope. Relative cell migration was determined by the mean number of migrated cells in eight random fields on the underside of the filter. For each experiment, three independent filters were analyzed.

Statistical analysis. Statistical analysis was performed using SPSS software, version 13.0 (SPSS, Inc., Chicago, IL, USA) and the data are expressed as the mean \pm standard deviation. Comparisons were conducted using Student's t-test. $\mathrm{P}<0.05$ was considered to indicate a statistically significant difference.

\section{Results}

Overexpression of survivin enhances colon carcinoma cell invasion. To investigate the potential role of survivin in cell 
A
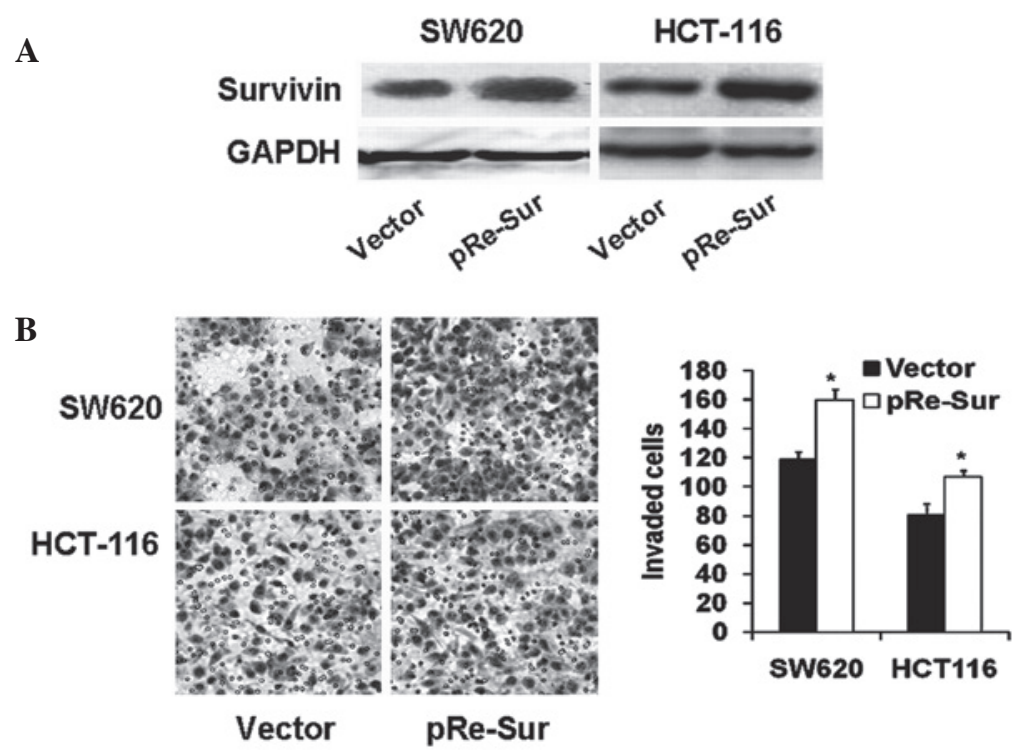

Figure 1. Survivin enhances invasion in human colon carcinoma cell lines, SW620 and HCT-116. (A) Human colon carcinoma SW620 and HCT-116 cells overexpressing survivin (pRe-Sur) were collected and survivin protein levels were analyzed by western blotting. GAPDH expression was the internal control for loading of protein. (B) Colon carcinoma cells were transfected with survivin control vector and survivin (pRe-Sur), and the motility of the cells was evaluated by using Boyden chamber assay. Original magnification, $\mathrm{x} 400$. Bars represent the mean $\pm \mathrm{SD}$. "P<0.01.pRe-Sur, pReceiver-survivin.

A

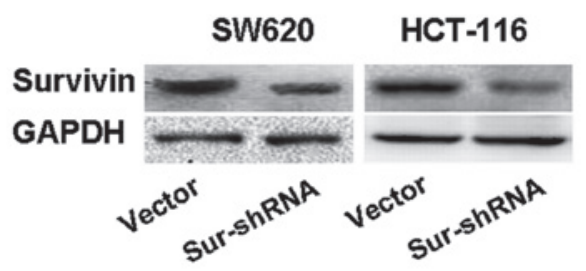

B
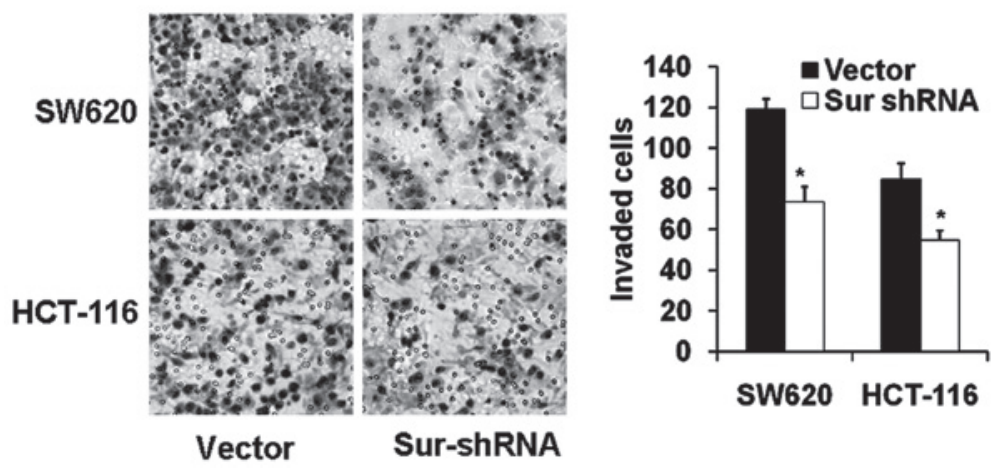

Figure 2. Survivin knockdown impairs invasion in human colon carcinoma cell lines, SW620 and HCT-116. Human colon carcinoma SW620 and HCT-116 cells were transfected with survivin shRNA (Sur-shRNA). (A) The cells were collected and survivin protein levels were analyzed by western blotting. GAPDH expression served as the internal control for loading of protein. (B) The motility of the cells was evaluated by using Boyden chamber assay. Original magnification, $\mathrm{x} 400$. Bars represent the mean $\pm \mathrm{SD}$. ${ }^{*} \mathrm{P}<0.01$. shRNA, short hairpin RNA.

invasion, cell invasion was examined in human colon carcinoma SW620 and HCT-116 cells overexpressing survivin. Western blot analysis confirmed the upregulation of survivin (Fig. 1A) and the Boyden chamber assay demonstrated that survivin overexpression significantly enhanced cell invasion accordingly (Fig. 1B; $\mathrm{P}<0.01$ ).

Knockdown of survivin impairs colon carcinoma cell invasion. To confirm the role of survivin in the invasion of colon carcinoma, RNAi-mediated knockdown of survivin was performed to suppress survivin in SW620 and HCT-116 cells.
As expected, survivin was silenced markedly, as revealed by the western blot analysis (Fig. 2A). As shown in Fig. 2B, survivin-knockdown cells showed significant reductions in cell invasion $(\mathrm{P}<0.01)$. These data suggest that survivin may be involved in the invasion of colon carcinoma cells.

Survivin regulates MMP-7 expression in colon carcinoma cells. To examine the effect of survivin on MMP-7 expression, ectopic expression and siRNA-based knockdown of survivin were performed in colon carcinoma SW620 and HCT-116 cells. Western blot analysis and immunofluorescence demonstrated 
A

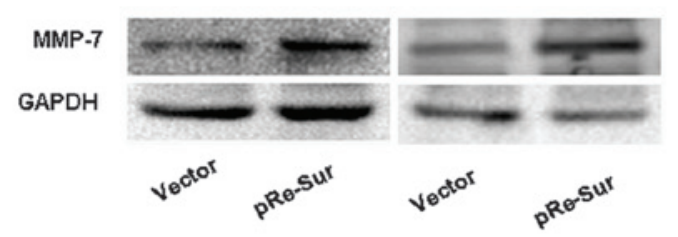

C

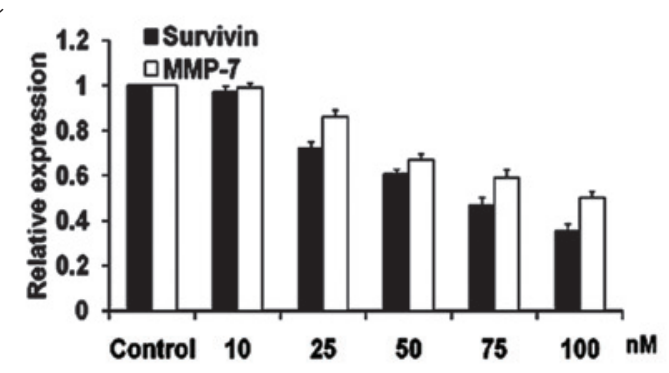

B

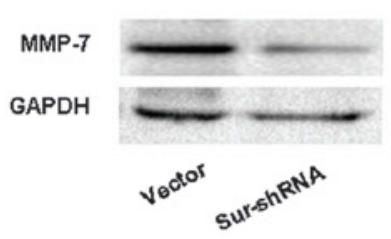

HCT-116

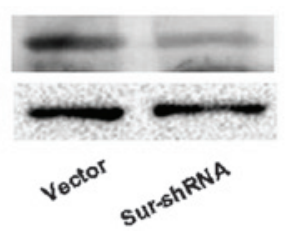

D

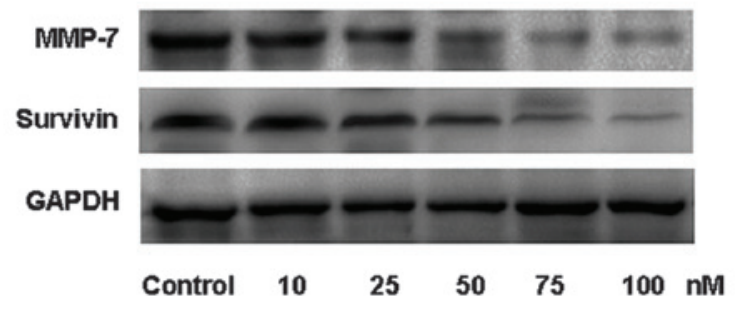

$\mathbf{E}$
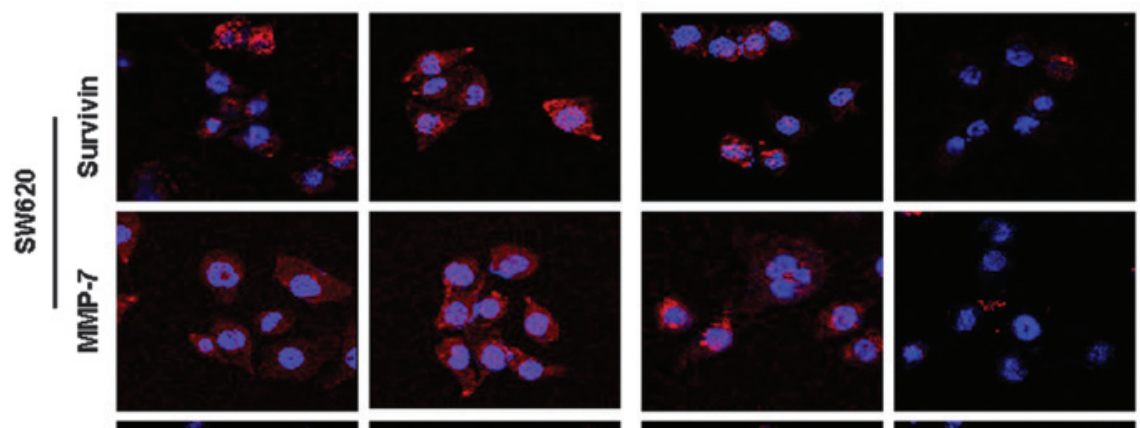

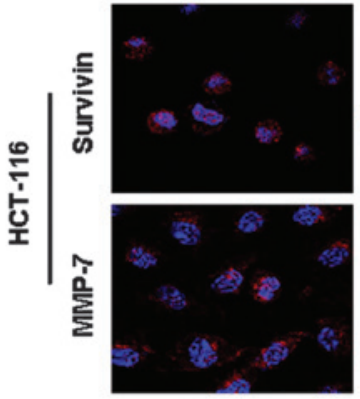

Vector

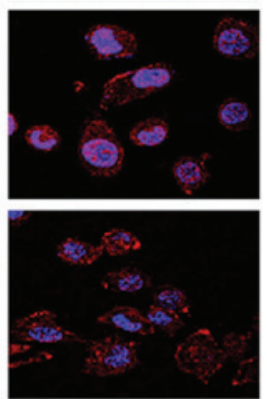

pRe-Sur

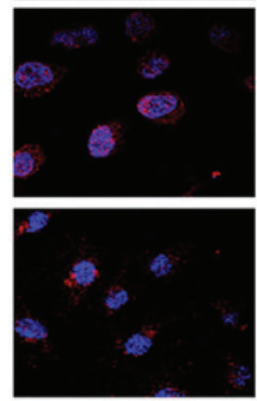

Vector

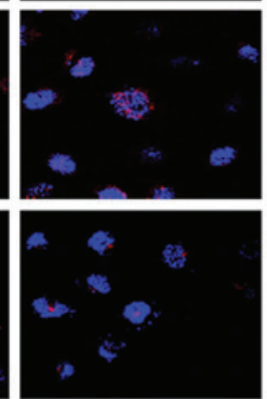

Sur-shRNA

Figure 3. MMP-7 expression in survivin- or survivin shRNA-transfected SW620 and HCT-116 cells. (A) Cells were collected and MMP-7 protein levels were analyzed by western blotting. Survivin increased MMP-7 activation in survivin-transfected cell lines. (B) Knockdown of survivin expression levels decreased MMP-7 expression levels in Sur-shRNA-transfected cell lines, as identified by western blotting. GAPDH expression was the internal control for loading of protein. Expression levels of MMP-7 after survivin siRNA infection at different doses (nM) in SW620 cells by (C) qPCR and (D) western blot analysis. (E) Immunofluorescence staining reveals increased expression levels of survivin and MMP-7 in survivin-overexpressing cells (pRe-Sur) and reduced expression levels in survivin-silenced cells (Sur-shRNA), in comparison with the vector controls. Original magnification, $\mathrm{x} 400$. MMP-7, metalloprotease-7; pRe-Sur, pReceiver-survivin; shRNA, short hairpin RNA; siRNA, small interfering RNA.

that the overexpression of survivin induced upregulation of MMP-7 (Fig. 3A and E). By contrast, MMP-7 expression levels were markedly suppressed in survivin-silenced colon carcinoma cells (Fig 3B and E). Furthermore, decreased expression levels of survivin led to a dose-dependent reduction in MMP-7 expression levels (Fig. 3C and D; P<0.01).

Role of MMP-7 in survivin-mediated invasion. To confirm the importance of MMP-7 in survivin-induced cell invasion, RNAi-mediated silencing of MMP-7 was performed in SW620 and HCT-116 cells and resulted in effective reduction of the endogenous MMP-7, as determined by qPCR (Fig. 4A). Notably the optimum reduction required $48 \mathrm{~h}$ of RNAi-mediated silencing. Subsequently, the protein expression of survivin and MMP-7 was assessed following cell culture for $48 \mathrm{~h}$. As shown in Fig. 4B, MMP-7 expression levels were reduced successfully using RNAi, as revealed by western blot analysis. Notably, the knockdown of MMP-7 did not alter the expression levels of survivin in colon carcinoma cells in comparison with the controls. Compared with the controls, reduced MMP-7 expression levels in the colon carcinoma cells significantly decreased the cell capacity for 
A
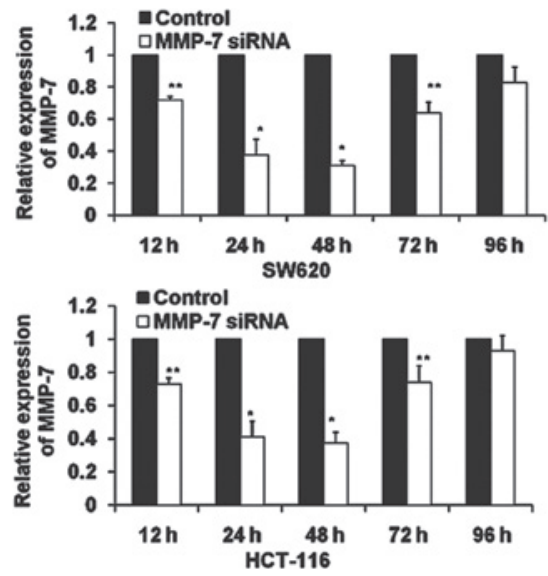

B

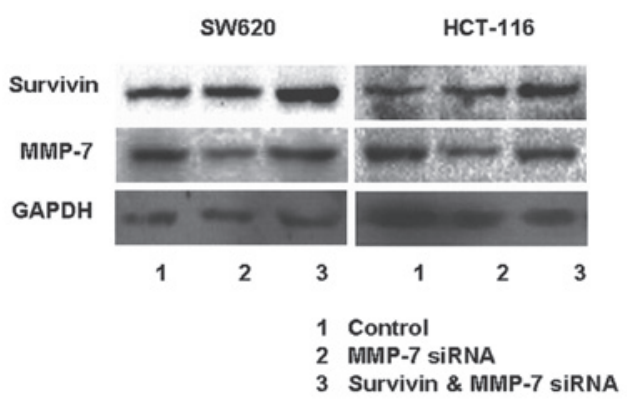

C
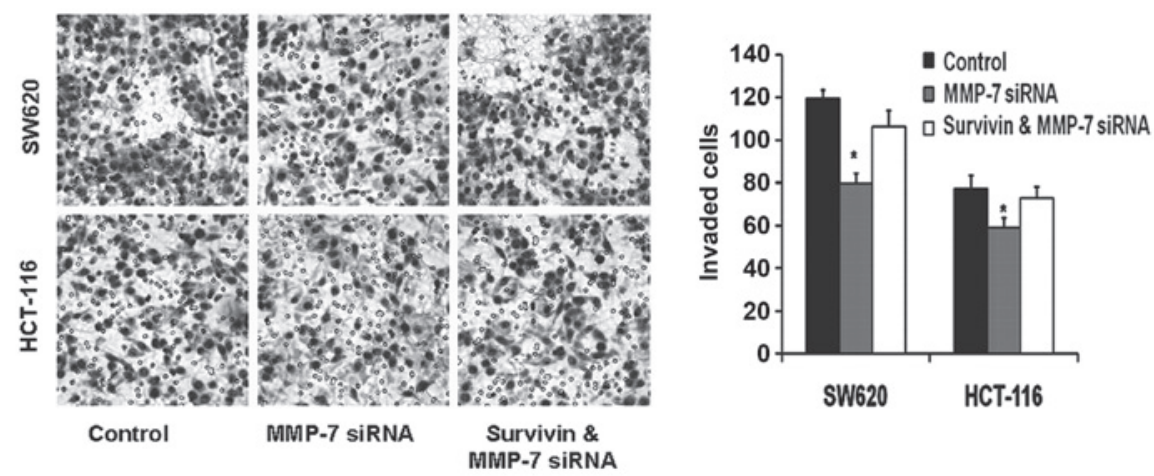

Figure 4. Role of MMP-7 in the survivin promotion of colon carcinoma cell motility. (A) Colon carcinoma cells were transfected with scrambled siRNA (the control) or MMP-7-specific siRNA, then cells were collected after 12, 24, 48, 72 and 96 h and MMP-7 mRNA levels were analyzed by qPCR. (B) Survivin and MMP-7 protein levels were analyzed in the colon carcinoma cells and in the cells overexpressing survivin by using western blotting $48 \mathrm{~h}$ after transfection with MMP-7 siRNA. (C) Silencing MMP-7 expression in colon carcinoma cells reduced their motility ability, as evaluated by Boyden chamber assay. However, decreased MMP-7 expression levels did not correlate with significant changes in the motility ability of cells overexpressing survivin. ${ }^{*} \mathrm{P}<0.01$ compared with the control. MMP-7, metalloproteinase-7; siRNA, small interfering RNA.

invasion, as evaluated by Boyden chamber assay $(\mathrm{P}<0.01)$ However, no significant difference was observed in the cells also overexpressing survivin ( $\mathrm{P} \geq 0.05)$ (Fig. 4C).

\section{Discussion}

CRC is the third most common type of cancer worldwide. The majority of individuals who are diagnosed with this disease are already at an advanced stage (20). Despite significant advances in early diagnosis and treatment, the survival rate of patients has not yet markedly improved, which is mainly due to the high rate of localized recurrence and distant metastasis following surgical resection (21). The exact mechanisms underlying the aggressive progression of CRC remain to be clarified. Therefore, elucidating the underlying mechanisms that drive CRC cell invasion and metastasis may aid in the exploitation of strategies for effective CRC treatment.

Survivin is the smallest member of the IAP family of anti-apoptotic proteins. It is highly expressed in tumors and is thought to promote tumor progression through apoptotic inhibition and mitotic chromosomal alignment $(1,22)$. Studies have demonstrated that overexpression of survivin is correlated with increased invasion and metastasis of human cancer, such as melanoma, prostate cancer, breast cancer and endometrial carcinoma $(2-4,23)$. Similar to these observations, the present study, to the best of our knowledge, has demonstrated for the first time that the overexpression of survivin is capable of enhancing cell invasion in the SW620 and HCT-116 colon carcinoma cell lines. Studies performed with the use of chemically synthesized siRNAs or plasmid/viral vectors encoding shRNAs have suggested that RNAi-mediated survivin knockdown is able to suppress cancer cell proliferation and promote caspase-dependent apoptosis in a number of human tumor cell models (24). Studies from our laboratory and findings of other studies have demonstrated that inhibition of survivin using shRNA and siRNA influences the biological features of colorectal carcinoma cells and inhibits invasion and metastasis of the human colon carcinoma cell line SW480 in vitro $(5,25)$. Similar to these observations, the present study found that RNAi-mediated knockdown of survivin is capable of significantly impairing cell invasion in SW620 and HCT-116 colon carcinoma cells. Therefore, through two methods of upregulating and inhibiting the expression of survivin in CRC cells, the results of the present study strongly indicate that survivin is required for tumor invasion in CRC. Notably, it has been demonstrated that inhibition of survivin is an effective therapeutic strategy in preclinical and clinical studies (26). The aforementioned studies suggest that survivin may be used as a potential target for cancer treatment in CRC patients. 
Studies have revealed that survivin enhances cell proliferation and suppresses apoptosis through a number of pathways $(27,28)$. The exact mechanisms by which survivin exerts this effect on the invasion ability of CRC remain largely unknown. In the present study, overexpression of survivin enhanced cell invasion and resulted in increased MMP-7 activation in SW620 and HCT-116 colon carcinoma cells. Conversely, knockdown of survivin using RNAi decreased MMP-7 expression levels. Notably, decreased expression levels of survivin led to a dose-dependent decrease in MMP-7 expression levels. However, knockdown of MMP-7 expression did not affect the expression levels of survivin in colon carcinoma cells compared with that in the controls. Similarly, Chu et al demonstrated that survivin knockdown downregulates the expression of MMPs in other types of colon carcinoma cell (6). Futhermore, the present study includes the primary investigation of the role of MMP-7 in cell invasion induced by survivin. Silencing of MMP-7 expression in the colon carcinoma cells reduced the cell invasion ability. By contrast, MMP-7 knockdown in transfected-survivin colon carcinoma cells did not contribute to significant changes in cell invasion. Overall, these results demonstrate that survivin may promote the invasion of colon carcinoma cells through the regulation of MMP-7 expression.

In conclusion, these data strongly suggest that upregulation of MMP-7 expression levels by survivin contributes to cell invasion, and that MMP-7 is a putative therapeutic target in the invasive progression of colon carcinoma. Additonal investigation is necessary to elucidate the regulatory mechanism between survivin and MMP-7.

\section{Acknowledgements}

This study was supported by funding from the National Natural Science Foundation of China (grant no. 81202125).

\section{References}

1. Mita AC, Mita MM, Nawrocki ST and Giles FJ: Survivin: key regulator of mitosis and apoptosis and novel target for cancer therapeutics. Clin Cancer Res 14: 5000-5005, 2008.

2. McKenzie JA, Liu T, Goodson AG and Grossman D: Survivin enhances motility of melanoma cells by supporting Akt activation and $\alpha 5$ integrin upregulation. Cancer Res 70: 7927-7937, 2010.

3. Zhang M, Coen JJ, Suzuki Y, et al: Survivin is a potential mediator of prostate cancer metastasis. Int J Radiat Oncol Biol Phys 78: 1095-1103, 2010.

4. Abdraboh ME, Gaur RL, Hollenbach AD, Sandquist D, Raj MH and Ouhtit A: Survivin is a novel target of CD44-promoted breast tumor invasion. Am J Pathol 179: 555-563, 2011.

5. Zhonghong L, Lianjie L, Changqing Z, Ying H, Yu J and Yan L: The influence of survivin shRNA on the cell cycle and the invasion of SW480 cells of colorectal carcinoma. J Exp Clin Cancer Res 27: 20, 2008.

6. Chu XY, Chen LB, Wang JH, et al: Overexpression of survivin is correlated with increased invasion and metastasis of colorectal cancer. J Surg Oncol 105: 520-528. 2012.
7. Chen P and Parks WC: Role of matrix metalloproteinases in epithelial migration. J Cell Biochem 108: 1233-1243, 2009.

8. Ii M, Yamamoto H, Adachi Y, Maruyama Y and Shinomura Y: Role of matrix metalloproteinase-7 (matrilysin) in human cancer invasion, apoptosis, growth, and angiogenesis. Exp Biol Med (Maywood) 231: 20-27, 2006

9. Wang FQ, So J, Reierstad S and Fishman DA: Matrilysin (MMP-7) promotes invasion of ovarian cancer cells by activation of progelatinase. Int J Cancer 114: 19-31, 2005

10. Lynch CC, Hikosaka A, Acuff HB, et al: MMP-7 promotes prostate cancer-induced osteolysis via the solubilization of RANKL. Cancer Cell 7: 485-496, 2005.

11. Shi M, Liu D, Duan H, et al: Catecholamine up-regulates MMP-7 expression by activating AP-1 and STAT3 in gastric cancer. Mol Cancer 9: 269, 2010

12. Sizemore ST and Keri RA: The forkhead box transcription factor FOXC1 promotes breast cancer invasion by inducing matrix metalloprotease 7 (MMP7) expression. J Biol Chem 287: 24631-24640, 2012.

13. Miyamoto S, Yano K, Sugimoto S, et al: Matrix metalloproteinase-7 facilitates insulin-like growth factor bioavailability through its proteinase activity on insulin-like growth factor binding protein 3. Cancer Res 64: 665-671, 2004.

14. Ho BY, Wu YM, Chang KJ and Pan TM: Dimerumic acid inhibits SW620 cell invasion by attenuating $\mathrm{H}_{2} \mathrm{O}_{2}$-mediated MMP-7 expression via JNK/C-Jun and ERK/C-Fos activation in an AP-1-dependent manner. Int J Biol Sci 7: 869-880, 2011.

15. Lee SK, Han YM, Yun J, et al: Phosphatase of regenerating liver-3 promotes migration and invasion by upregulating matrix metalloproteinases-7 in human colorectal cancer cells. Int J Cancer 131: E190-E203, 2012.

16. Wang WS, Chen PM, Wang HS, Liang WY and Su Y: Matrix metalloproteinase-7 increases resistance to Fas-mediated apoptosis and is a poor prognostic factor of patients with colorectal carcinoma. Carcinogenesis 27: 1113-1120, 2006.

17. Koskensalo S, Louhimo J, Nordling S, Hagström J and Haglund C: MMP-7 as a prognostic marker in colorectal cancer. Tumour Biol 32: 259-264, 2011.

18. Fang YJ, Lu ZH, Wang GQ, et al: Elevated expressions of MMP7, TROP2, and survivin are associated with survival, disease recurrence, and liver metastasis of colon cancer. Int J Colorectal Dis 24: 875-884, 2009.

19. Wang GQ, Lu ZH, Fang YJ, Chen G, Zhou ZW, Pan ZZ and Wan DS: Expression and clinical significance of survivin and matrix metalloproteinase-7 in colon cancer. Ai Zheng 28: 945-949, 2009 (In Chinese).

20. Jemal A, Bray F, Center MM, Ferlay J, Ward E and Forman D: Global cancer statistics. CA Cancer J Clin 61: 69-90, 2011.

21. Kelley RK, Wang G and Venook AP: Biomarker use in colorectal cancer therapy. J Natl Compr Canc Netw 9:1293-1302, 2011.

22. Altieri DC: Survivin, cancer networks and pathway-directed drug discovery. Nat Rev Cancer 8: 61-70, 2008.

23. Erkanli S, Bolat F, Kayaselcuk F, Demirhan B and Kuscu E: COX-2 and survivin are overexpressed and positively correlated in endometrial carcinoma. Gynecol Oncol 104: 320-325, 2007.

24. Pennati M, Folini M and Zaffaroni N: Targeting survivin in cancer therapy: fulfilled promises and open questions. Carcinogenesis 28: 1133-1139, 2007.

25. Wu JB, Nan QZ, Ma GF, et al: Short hairpin RNA-mediated survivin gene silencing inhibits invasion and metastasis of human colon carcinoma cell line SW480 in vitro. Nan Fang Yi Ke Da Xue Xue Bao 27: 951-954, 2007 (In Chinese).

26. Church DN and Talbot DC: Survivin in solid tumors: rationale for development of inhibitors. Curr Oncol Rep 14: 120-128, 2012.

27. Altieri DC: Survivin, versatile modulation of cell division and apoptosis in cancer. Oncogene 22: 8581-8589, 2003.

28. Kelly RJ, Lopez-Chavez A, Citrin D, Janik JE and Morris JC: Impacting tumor cell-fate by targeting the inhibitor of apoptosis protein survivin. Mol Cancer 10: 35, 2011. 